\title{
Energy Efficiency Maximization of Dynamic CoMP-JT Algorithm in Dense Small Cell Networks
}

\author{
Xuefei Peng, Jiandong Li $(D$, and Yifei Xu \\ State Key Laboratory of Integrated Service Networks, Xidian University, Xian 710071, China \\ Correspondence should be addressed to Jiandong Li; jdli@xidian.edu.cn
}

Received 2 April 2018; Revised 13 June 2018; Accepted 21 June 2018; Published 12 July 2018

Academic Editor: Shunqing Zhang

Copyright (C) 2018 Xuefei Peng et al. This is an open access article distributed under the Creative Commons Attribution License, which permits unrestricted use, distribution, and reproduction in any medium, provided the original work is properly cited.

\begin{abstract}
We firstly formulate the energy efficiency (EE) maximization problem of joint user association and power allocation considering minimum data rate requirement of small cell users (SUEs) and maximum transmit power constraint of small cell base stations (SBSs), which is NP-hard. Then, we propose a dynamic coordinated multipoint joint transmission (CoMP-JT) algorithm to improve EE. In the first phase, SUEs are associated with the SBSs close to them to reduce the loss of power by the proposed user association algorithm, where the associated SBSs of each small cell user (SUE) form a dynamic CoMP-JT set. In the second phase, through the methods of fractional programming and successive convex approximation, we transform the EE maximization subproblem of power allocation for SBSs into a convex problem that can be solved by proposed power allocation optimization algorithm. Moreover, we show that the proposed solution has a much lower computational complexity than that of the optimal solution obtained by exhaustive search. Simulation results demonstrate that the proposed solution has a better performance.
\end{abstract}

\section{Introduction}

In future $5 \mathrm{G}$ wireless network, $\mathrm{EE}$ improvement is arisen as a challenging issue [1]. Therefore, how to promote EE is of great significance. With the explosive increasing data traffic demands of users in 5G wireless cellular network, one of the prospective solutions for satisfying the data rate requirement of users and improving EE is the deployment of low power, low cost, and small coverage range SBSs. Small cell tier is an integral part of $5 \mathrm{G}$ heterogeneous cellular network architecture that can provide more opportunities for users to connect to the networks close to them, which will decrease power consumption $[2,3]$.

However, due to the dense deployment and spectrum sharing of small cells, the interference among small cells becomes a key factor that influences EE of the network [4].

On the one hand, several recent works [5-7] have adopted scheduling schemes to mitigate interference in small cell networks. In [5], the authors studied the performance of different scheduling methods under distinct channel models with considering the intersite distance. In [6], the authors formulated a fast convergent speed algorithm to solve the energy-efficient multijob scheduling function. In [7], dynamic clustering framework of multicell scheduling based on graph was proposed to mitigate intercell interference in dense small cell networks, and channel-aware resource allocation was incorporated in the dynamic clustering framework to provide different levels of tunable quality of service. However, EE optimization for dynamic coordination among SBSs is not considered [5-7].

On the other hand, some studies have discussed multicell CoMP transmission (CoMP) [8-15] of cellular network. CoMP-JT is a promising technique that guarantees data availability at multiple coordinated SBSs transmitted to a user simultaneously to improve the received signal quality of the user and reduce intercell interference, which is proposed in Third Generation Partnership Project (3GPP) LTE-Advanced systems [8]. In [9-11], the authors studied CoMP-JT from the aspects of throughput and coverage probability. However, EE optimization is not considered. In [12], the authors proposed two algorithms to tackle the problem of minimizing backhaul user data transfer by establishing CoMP joint processing beamforming matrix of multicell. In $[13,14]$, the authors formulated EE maximization problem with global precoding 


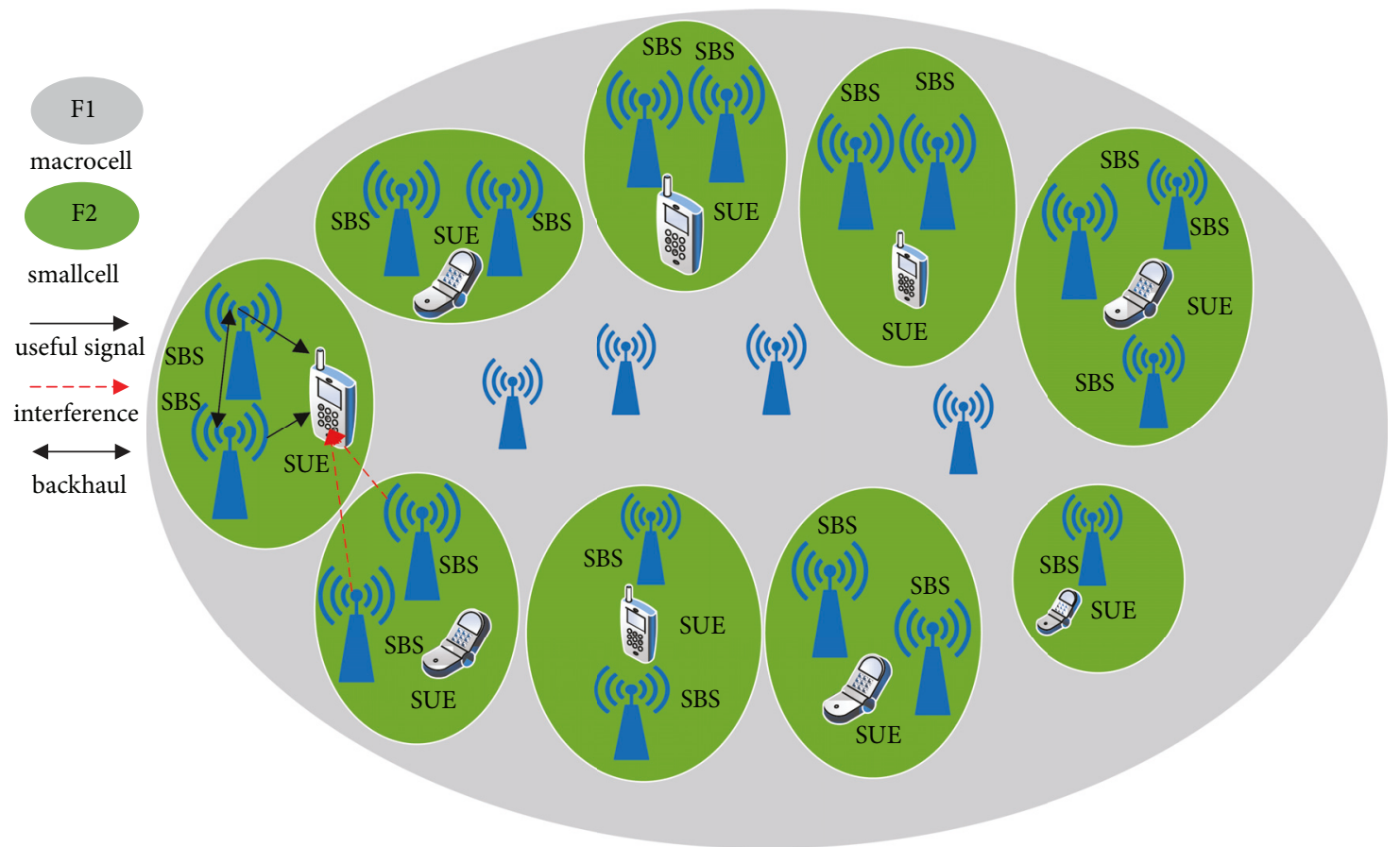

FIGURE 1: Network model.

matrix design. However, the beamforming and precoding matrix of global optimization are complex and difficult to realize in practical systems [12-14]. In [15], the authors proposed a distributed algorithm to solve the weighted EE maxmin fairness problem for CoMP systems. However, dynamic coordinated transmission among SBSs is not considered.

Unlike existing literature, a dynamic CoMP-JT algorithm considering user association, power allocation, and minimum data rate requirement of SUEs is proposed in this paper to mitigate interference and maximize EE.

The main contributions of this paper are summarized as follows:

(i) We utilize dynamic CoMP-JT as an interference management technique to mitigate interference and improve $\mathrm{EE}$, where minimum data rate requirements of SUEs and maximum transmit power constraint of SBSs are considered.

(ii) We propose to solve the NP-hard problem of EE maximization through the proposed dynamic CoMP-JT algorithm. In the first phase, each SUE is associated with SBSs close to them to reduce the loss of power by the proposed user association algorithm. In the second phase, we transform the formulated EE optimization problem into a convex problem by methods of fractional programming and successive convex approximation. Finally, we solve the formulated problem by the proposed power allocation optimization algorithm.

(iii) We analyze the computational complexities of the optimal solution and the proposed solution and compare the performance of near-optimal solution, no-CoMP, and random-CoMP with the proposed solution.

The rest of this paper organized as follows. The system model and problem formulation are given in Section 2. In Section 3, the dynamic CoMP-JT algorithm is proposed. In
Section 4, numerical results are given. Finally, the paper is concluded in Section 5.

\section{System Model and Problem Formulation}

Our system model is depicted in Figure 1. We consider a downlink orthogonal frequency division multiple access (OFDMA) network, where macrocell tier and small cell tier are allocated with orthogonal spectrums. Therefore, there is no cross-tier interference and we focus on the EE analysis of small cell tier, where only the interference between SBSs and SUEs is considered. Assume that SUEs are randomly and uniformly distributed in the coverage region of SBSs. Let $S=\{1,2 \cdots, S\}$ denote the set of all SBSs with low transmit power and $U=\{1,2, \cdots U\}$ denote the set of SUEs. We will next derive the optimization problem of EE, where the EE is defined as the transmitted bits per unit energy consumption and equals the ratio of the sum data rate to the total power consumption. We define a $\mathrm{SU} \times 1$ vector $\mathbf{p}=\left(\mathbf{p}_{1}, \mathbf{p}_{2}, \cdots \mathbf{p}_{s} \cdots \mathbf{p}_{\mathrm{S}}\right)^{T}$ to represent the power of all SBSs allocate for all SUEs, where $\mathbf{p}_{s}=\left(p_{s 1}, p_{s 2} \cdots p_{s \mathrm{u}}\right)$ denotes the power of SBSs allocated for SUE 1 to SUE U. Let $\mathbf{x}$ denote a binary vector to represent the association relationship between SBSs and SUEs. Its element is an association indicator decision variable $x_{s u} \in\{0,1\}$ denoting whether SBS $s$ is associated with SUE $u, x_{s u}=1$, if SBS $s$ is associated with SUE $u$; otherwise $x_{s u}=0$.

From [16], we can derive the received signal strength of SUE $u$ associated with one or more SBSs as follows:

$$
p_{u}^{\mathrm{r}}=\sum_{s \in S}\left(g_{s u} \sqrt{x_{s u} p_{s u}}\right)^{2}=\sum_{s \in S} x_{s u} p_{s u} h_{s u}
$$


where $p_{s u}$ is SBSs transmit power for SUE $u$ and $g_{s u}$ is the channel gain from SBS $s$ to SUE $u$, which includes path loss, Rayleigh, and shadowing fading. Moreover, we assume that $h_{s u}=g_{s u}^{2}$.

Then, we can obtain the signal to interference and noise ratio (SINR) of SUE $u$ as follows:

$$
\Gamma_{u}(\mathbf{x}, \mathbf{p})=\frac{p_{u}^{\mathrm{r}}}{\sum_{l \in S, i \neq u}\left(x_{l i} p_{l u} h_{l u}\right)+\delta^{2}},
$$

where the first part of the denominator denotes the interference of SUE $u$ from the noncooperating SBSs. The second part of denominator $\delta^{2}$ is the power of additive white Gaussian noise. Furthermore, we can get the data rate of SUE $u$ as follows:

$$
R_{u}(\mathbf{x}, \mathbf{p})=\Delta f \log _{2}\left(1+\Gamma_{u}(\mathbf{x}, \mathbf{p})\right),
$$

where $\Delta f$ is the bandwidth of the network.

The power consumption of SUE $u$ can be also derived as

$$
P_{u}(\mathbf{x}, \mathbf{p})=x_{s u} p_{b c k}+\sum_{s \in S} x_{s u}\left(\Delta_{p} p_{s u}+p_{s u}^{c}\right),
$$

where $p_{b c k}$ is the power consumption of backhaul. $\Delta_{p}$ is a constant concerning the power amplifier efficiency and $p_{s u}^{c}$ denotes the circuit power consumption.

Then, we can formulate the EE maximization problem of joint user association and power allocation as follows:

$$
\begin{aligned}
& \max _{\mathbf{x}, \mathbf{p}} \quad \eta_{E E}=\frac{R_{t o t}(\mathbf{x}, \mathbf{p})}{P_{t o t}(\mathbf{x}, \mathbf{p})}=\frac{\sum_{u=1}^{\mathrm{U}} R_{u}(\mathbf{x}, \mathbf{p})}{\sum_{u=1}^{\mathrm{U}} P_{u}(\mathbf{x}, \mathbf{p})} \\
& \text { s.t. } \quad(5 a): R_{u} \geq R_{u}^{\min }, \quad \forall u \in U \\
& (5 b): \sum_{u=1}^{\mathrm{U}} p_{s u} \leq p_{s}^{\max }, \quad \forall s \in S \\
& (5 c): \sum_{u=1}^{\mathrm{U}} x_{s u} \leq 1, \quad \forall s \in S \\
& (5 d): x_{s u}=\{0,1\}, \quad \forall x_{s u} \in \mathbf{x},
\end{aligned}
$$

where (5a) specifies the minimum system data rate requirement $R_{u}^{\min }$ of SUE $u$. Equation (5b) is the individual maximum transmit power constraint $p_{s}^{\max }$ of SBS $s$. Equations $(5 \mathrm{c})$ and (5d) are CoMP-JT constraint, which guarantees that one SBS can only be associated with one SUE and one SUE can be served by one or more SBSs.

Owing to the NP-hardness of joint user association and power allocation, obtaining its optimal solution by exhaustive scheme will incur unaffordable computational complexity $[17,18]$. Therefore, we propose a dynamic CoMP-JT algorithm to solve problem (5).

\section{Dynamic COMP-JT Algorithm}

The proposed dynamic CoMP-JT algorithm consists of two phases, which are user association forming the CoMP-JT set and power allocation optimization.
3.1. Proposed User Association Algorithm. In this phase, we will determine the association relationship between SBSs and SUEs by the proposed user association algorithm.

According to the path loss from SBSs to SUEs, we firstly determine the size and the set of CoMP-JT SBSs for each SUE. Let $M_{u}$ denote the set of associated SBSs of SUE $u$ and $S^{\prime}$ denote the set of SBSs that has not been chosen by other SUEs. In each round, we add the SBS with the minimum path loss for SUE $u$ from the set $S^{\prime}$ to the set of associated SBSs of SUE $u$ according to the principle $M_{u}=M_{u} \cup \arg \min _{s \in S^{\prime}}\left\{\mathrm{PL}_{s u}\right\}$, where the path loss from SBS $s$ to SUE $u$ is $\mathrm{PL}_{s u}$ in our model. We will repeat the above procedure until $S^{\prime}=\phi$. Since SBSs are densely deployed in our considered scenario, therefore, each SUE can be associated with at least one SBS in our proposed user association algorithm. Moreover, SUEs can be associated with the SBSs close to them because the path losses between SBSs and SUEs are small in dense small cell networks. The proposed user association algorithm ensures that SUEs associated with the SBSs near them are within the communication range through the condition $\mathrm{PL}_{s u}<\mathrm{PL}_{0}$; i.e., SBSs and SUEs should be matched with each other through the bidirectional selection method that SUEs choose their serving SBSs; meanwhile SBSs choose the associated SUEs in their coverage range. To further reduce path loss and save power consumption, we traverse and find all the possible CoMP-JT user association cases through polling all the orders that SUEs choose their associated SBSs. The detail description of the proposed user algorithm is shown in Algorithm 1.

3.2. Power Allocation Optimization. In this phase, we will optimize power allocation of SBSs to improve EE. According to the established association relationship between SBSs and SUEs of the former phase, the EE maximization problem of the network with CoMP-JT can be transformed as follows:

$$
\begin{array}{ll}
\max _{\mathbf{p}} & \eta_{E E}=\frac{R_{\text {tot }}(\mathbf{p})}{P_{\text {tot }}(\mathbf{p})}=\frac{\sum_{u=1}^{\mathrm{U}} R_{u}(\mathbf{p})}{\sum_{u=1}^{\mathrm{U}} p_{u}(\mathbf{p})} \\
\text { s.t. } \quad(6 a): R_{u} \geq R_{u}^{\min }, \quad \forall u \in U \\
\\
(6 b): \sum_{u=1}^{\mathrm{U}} p_{s u} \leq p_{s}^{\max }, \quad \forall s \in S .
\end{array}
$$

Compared (6) with (5), we can observe that the EE maximization problem in (6) is determined by the power allocation vector of SBSs since user association vector $\mathbf{x}$ has been determined in the proposed user association algorithm.

As the problem in $[13,14,19]$, the optimization problem (6) with an objective function in fractional form, we use the method of fractional programming that equivalently convert the objective function to subtractive form as follows:

$$
\begin{array}{ll}
\max _{\mathbf{p}} & R_{\text {tot }}(\mathbf{p})-\eta_{E E}^{n} P_{\text {tot }}(\mathbf{p}) \\
\text { s.t. } & (6 a),(6 b) .
\end{array}
$$

The objective function in (7) can be transformed as

$$
R_{t o t}(\mathbf{p})-\eta_{E E}^{n} P_{t o t}(\mathbf{p})=g(\mathbf{p})-r(\mathbf{p}),
$$




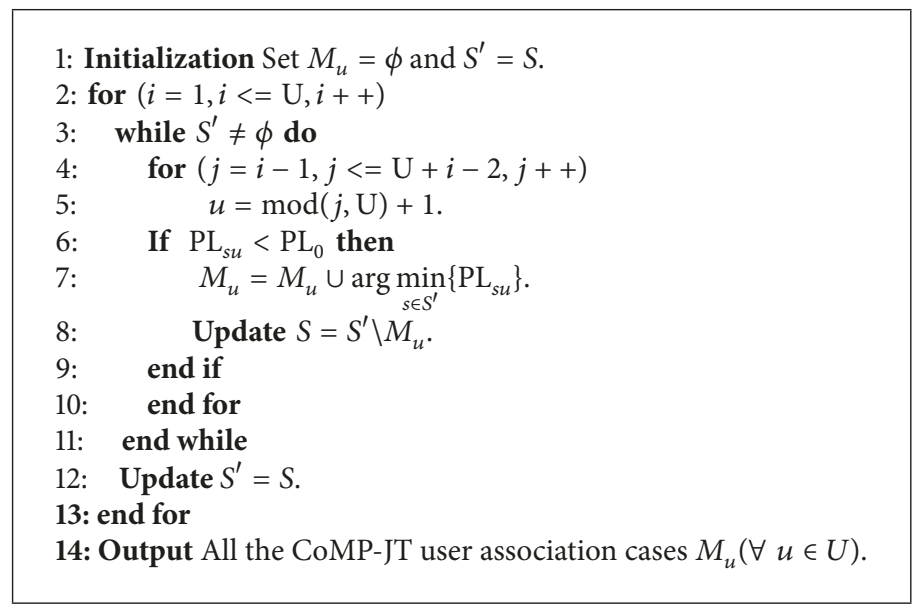

Algorithm 1: Proposed user association algorithm.

where

$$
\begin{aligned}
g(\mathbf{p})= & \Delta f \sum_{u=1}^{\mathrm{U}} \log _{2}\left(\sum_{s=1}^{\mathrm{S}} p_{s u} h_{s u}+\delta^{2}\right)-\eta_{E E}^{n} \\
& \times \sum_{u=1}^{U} P_{u}(\mathbf{p})
\end{aligned}
$$

and

$$
r(\mathbf{p})=\Delta f \sum_{u=1}^{\mathrm{U}} \log _{2}\left(\sum_{l=1, l \notin M_{u}}^{\mathrm{S}} p_{l u} h_{l u}+\delta^{2}\right) .
$$

Since (6a) is nonconvex constraint, we rearrange (6a) in (7) and an equivalent convex linear form $\left(6 \mathrm{a}^{\prime}\right)$ can be derived as follows:

$$
\begin{gathered}
\left(6 a^{\prime}\right): \sum_{s \in M_{u}} p_{s u} h_{s u}+\left(1-2^{\left(R_{u}^{\min } / \Delta f\right)}\right) \\
\times\left(\sum_{l=1, l \notin M_{u}}^{S} p_{l} h_{l u}+\delta^{2}\right) \geq 0 .
\end{gathered}
$$

Hence, optimization problem (7) is equivalent to

$$
\begin{gathered}
\max _{\mathbf{p}} g(\mathbf{p})-r(\mathbf{p}) \\
\text { s.t }\left(6 a^{\prime}\right),(6 b) .
\end{gathered}
$$

Therefore, the objective $g(\mathbf{p})-r(\mathbf{p})$ in (12) is the difference between two concave functions program. The gradient of $r(\mathbf{p})$ at power $\mathbf{p}$ is given by

$$
\nabla r(\mathbf{p})=\Delta f \sum_{u=1}^{\mathrm{U}} \frac{1}{\sum_{l=1, l \notin M_{u}}^{\mathrm{S}} p_{l u} h_{l u}+\delta^{2}} \mathbf{v}_{u}
$$

where $\mathbf{v}_{u}(z)=0$ and $z \in M_{u} ; \mathbf{v}_{u}(l)=h_{l u} / \ln 2, l \notin M_{u}$. Then, we adopt successive convex approximation method to approximate $r(\mathbf{p})$ by its tangent function $r(\mathbf{p}) \approx r\left(\mathbf{p}^{n}\right)+$ $\nabla r^{T}\left(\mathbf{p}^{n}\right)\left(\mathbf{p}-\mathbf{p}^{n}\right)$ at each step; we can obtain the optimization problem as follows:

$$
\begin{array}{ll}
\max _{\mathbf{p}} & g(\mathbf{p})-\left(r\left(\mathbf{p}^{n}\right)+\nabla r^{T}\left(\mathbf{p}^{n}\right)\left(\mathbf{p}-\mathbf{p}^{n}\right)\right) \\
\text { s.t. } & \left(6 a^{\prime}\right),(6 b) .
\end{array}
$$

Now, (14) is a standard convex optimization problem, and interior point method in [20] can be applied to solve it.

We transverse all the possible CoMP-JT user association cases obtained in Algorithm 1 and further get optimal EE solution for each user association case through power allocation optimization Algorithm 2. Finally, we can find the maximization value of $\mathrm{EE}$ in all the possible user association cases. The detail description of power allocation optimization process is shown in Algorithm 2.

3.3. Complexity Analysis. In this section, we analyze the computational complexities of the optimal and the proposed solutions. Finding optimal solution needs to exhaust all the association cases. Thus the computational complexity of optimal association is $\mathrm{O}\left(\mathrm{S} ! /\left(\left(\mathrm{S}-\sum_{u=1}^{\mathrm{U}} w_{u}\right) \times \mathrm{U} ! \times w_{u} !\right)\right)$, where $w_{u}$ denotes the number of associated SBSs of SUE $u$. However, Algorithm 1 firstly chooses the SBSs in $S^{\prime}$ with the minimum path loss for SUEs, which requires a computational complexity $\mathrm{O}\left(\mathrm{C}_{\mathrm{S}}^{\mathrm{U}}\right)$. Then, Algorithm 1 needs to repeat the above procedure until $S^{\prime}=\phi$, which requires a computational complexity not larger than $\mathrm{O}\left(\mathrm{C}_{\mathrm{S}-(k-1) \mathrm{U}}^{\mathrm{U}}\right)$ in the $k$ round. Moreover, Algorithm 1 needs to transverse all the user association cases in outer circulation with the computational complexity $\mathrm{U}$. Therefore, Algorithm 1 requires a computational complexity no larger than $O\left(U *\left(\mathrm{C}_{\mathrm{S}}^{\mathrm{U}}+\cdots+\mathrm{C}_{\mathrm{S}-(k-1) \mathrm{U}}^{\mathrm{U}}\right)\right)$, which is much lower than that of the optimal scheme. Suppose that the maximum outer power iterative of interior point scheme is $\mathrm{N}_{\max }$ and the computational complexity of applying the successive convex approximation scheme depends on the outer iteration and iterative solution of each step; thus the overall computational complexity of interior point scheme is $\mathrm{O}\left(\mathrm{N}_{\max } \mathrm{SU}\right)$. So the overall computational complexity of 


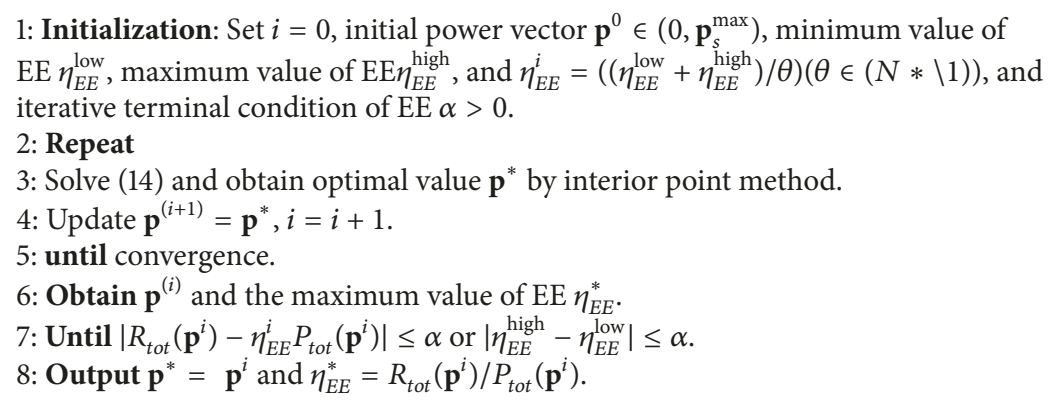

Algorithm 2: Power allocation optimization algorithm.

our proposed solution is $\mathrm{O}\left(\mathrm{N}_{\max } \mathrm{SU}^{2}\left(\mathrm{C}_{\mathrm{S}}^{\mathrm{U}}+\cdots+\mathrm{C}_{\mathrm{S}-(k-1) \mathrm{U}}^{\mathrm{U}}\right)\right)$. However, we need to traverse all the power values for the optimal solution, which has a complexity of $\mathrm{Q}^{\mathrm{U}}$, where $\mathrm{Q}$ is the number of quantified power allocation values for each SUE of a SBS. So the overall computational complexity of the optimal solution is $\mathrm{O}\left(\mathrm{Q}^{\mathrm{U}} \mathrm{S} ! /\left(\left(\mathrm{S}-\sum_{u=1}^{\mathrm{U}} w_{u}\right) \times \mathrm{U} ! \times w_{u} !\right)\right)$. Consequently, our proposed solution has a notably reduced complexity compared with the optimal solution.

\section{Simulation Results}

In our simulation, all the SUEs and SBSs are randomly and uniformly distributed in the area of $40 \mathrm{~m} * 40 \mathrm{~m}$. We consider that the channel model includes path loss, Rayleigh, and shadowing fading, where the path loss from SBS $s$ to SUE $u$ is given by $\mathrm{PL}_{s u}=38.46+20 \times \log 10\left(\mathrm{~d}_{s u}\right)$, where $\mathrm{d}_{s u}$ denotes the distance between SBS $s$ and SUE $u$, with the unit $m$. The shadowing standard deviations are $8 \mathrm{~dB}$ for the link between SBS and the SUE [21]. Besides, $\mathrm{PL}_{0}=58.46 \mathrm{~dB}$, which is calculated by assuming the coverage radius of each SBS is 10 $m$.

Figure 2 plots EE with respect to the iteration number under different maximum transmit powers of SBSs, and Figure 3 plots $g(\mathbf{p})-r(\mathbf{p})$ with respect to the iteration number under different initial iterative transmit power values. We can observe that the proposed dynamic COMP-JT Algorithm can quickly converge to the stable EE maximization solution, which confirms the fact that the proposed solution has a considerable convergence property.

Next, we compare the proposed solution with the following solutions:

(i) Near-optimal solution: Algorithm 1 is adopted as the user association scheme. Moreover, since the power allocation vector is continuous, therefore, we firstly equally quantify each power allocation variable into 20 values in $\left[0, p_{s}^{\max }\right]$ and then we need to exhaustively traverse the quantified $20^{\mathrm{U}}$ values into the objective function to obtain near-optimal solution.

(ii) SecondNear-CoMP [16]: We assume that system bandwidth is shared by all the SUEs, and SUEs are associated with the nearest SBS and the SBS (except the serving SBS) nearest to the SUE from the set of SBSs which has not been chosen by other SUEs as their coordinated multipoint joint

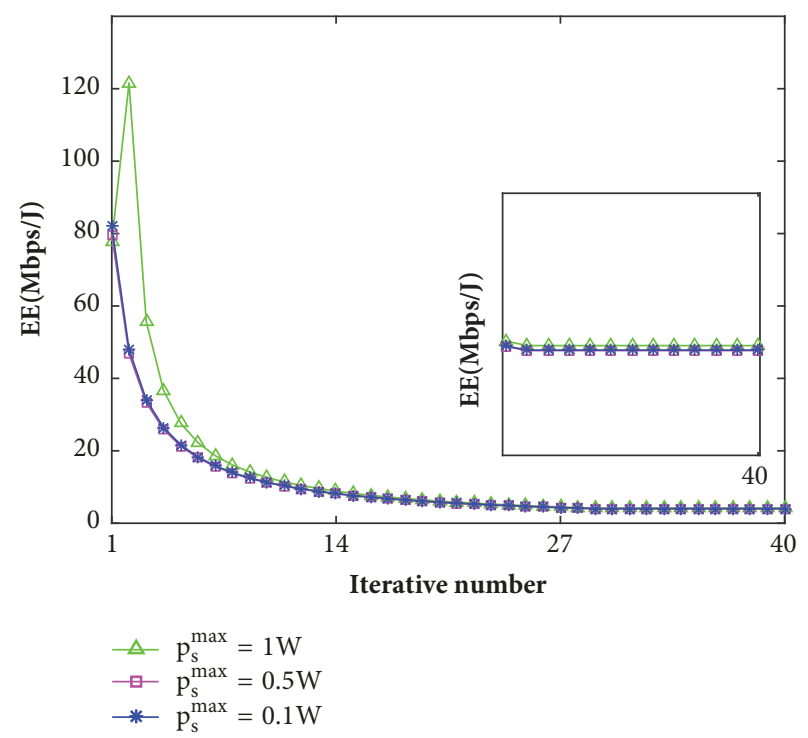

Figure 2: EE vs. iterative number.

transmission (CoMP-JT) SBSs. Moreover, Algorithm 2 is adopted as the power allocation optimization algorithm. We call this method SecondNear-CoMP solution.

(iii) No-CoMP [19]: Each SUE is associated with the nearest SBS that has not been associated with other SUEs. Moreover, Algorithm 2 is adopted as the power allocation optimization algorithm.

(iv) Random-CoMP: Each SUE is randomly associated with the SBSs close to it as Algorithm 1. Besides, Algorithm 2 is adopted as the power allocation optimization algorithm.

Figure 4 shows the EE with respect to different data rate requirement of SUEs, where $p_{s}^{\max }=1 \mathrm{w}$ and the number of SUEs and SBSs is 16 and 22, respectively. Since obtaining the optimal solution of problem (5) by exhaustive search will incur prohibitive computational complexity in dense small cell network, thus, an efficient near-optimal solution of power allocation is used in (5), where 20 uniformly quantified power allocation values for each SUE of a SBS are traversed. We can observe the performance gap between the proposed and the optimal solution becomes a little larger when $R_{u}^{\min }$ 


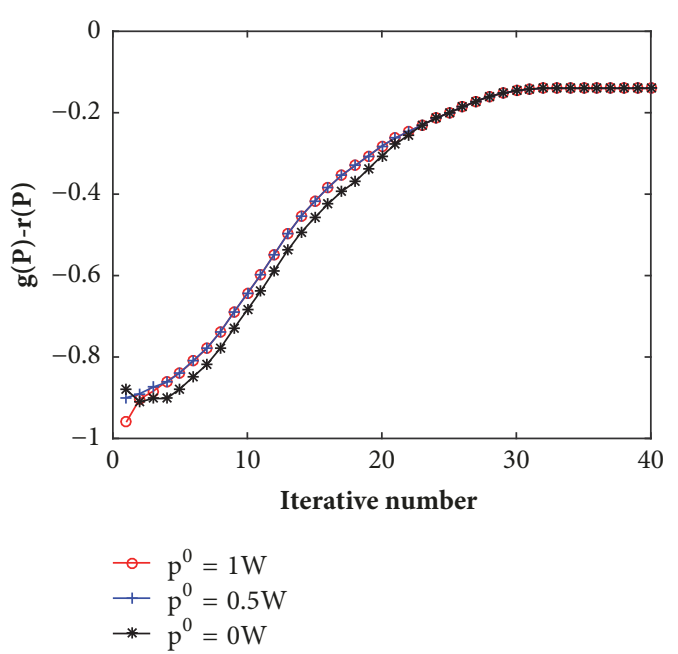

Figure 3: $g(\mathbf{p})-r(\mathbf{p})$ vs. iterative number.

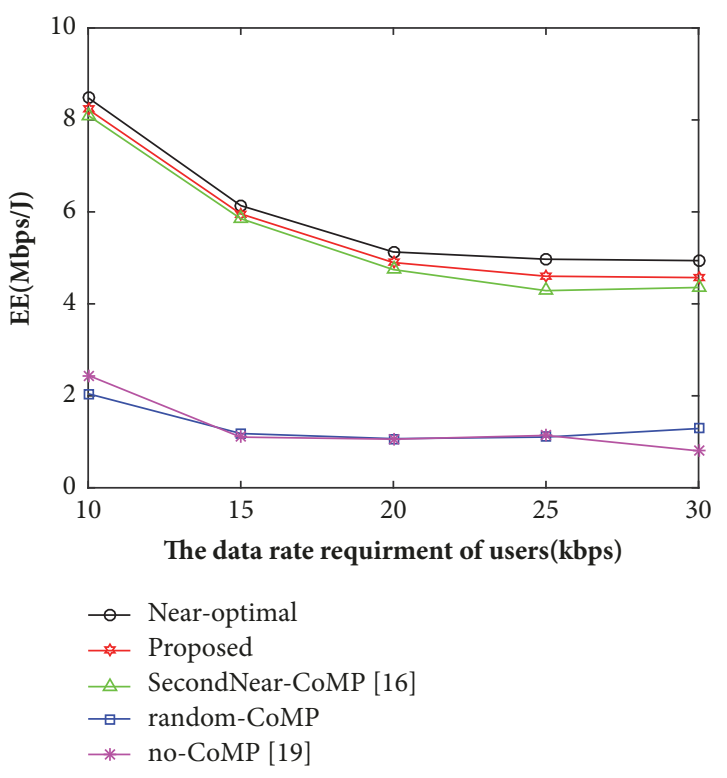

FIgURE 4: EE vs. $R_{u}^{\mathrm{min}}$.

increases. Nevertheless, the proposed solution has a significantly reduced computational complexity. Besides, we can intuitively observe that the proposed CoMP-JT scheme has a better performance than the other three schemes and random CoMP-JT scheme has a better performance than transmission without CoMP when $R_{u}^{\mathrm{min}}$ becomes larger. The reason is that the proposed CoMP-JT ensures that SUEs are associated near SBSs with lower path losses. Moreover, CoMP-JT schemes can satisfy higher $R_{u}^{\min }$ by exploiting interference through constructing CoMP-JT set.

Figure 5 depicts the EE with respect to different number of SBSs, where $p_{s}^{\max }=1 \mathrm{w}, R_{u}^{\mathrm{min}}=20 \mathrm{kbps}$, and the number of SUEs is 16 . We can observe the performance gap between the proposed and the optimal solution becomes a little larger with the increasing number of SBSs. The reason is

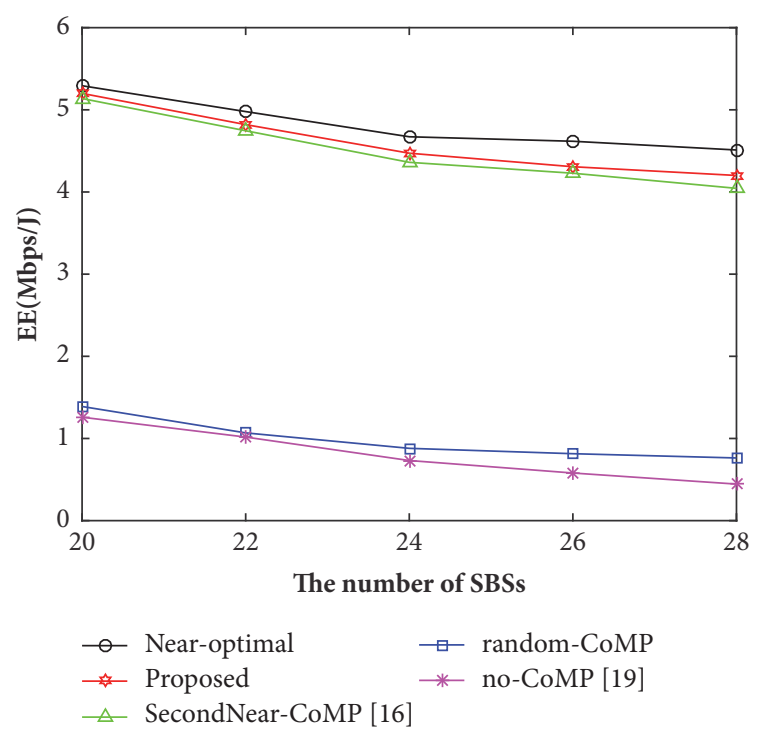

FIgUre 5: EE vs. The number of SBSs.

that computational complexity increases with the number of deployed SBSs. Besides, we can observe that the performance of our proposed CoMP-JT scheme is better than the other three schemes. The reason is that our proposed scheme guarantees that SUEs are associated with SBSs closer to them, which will reduce the loss of power. Moreover, the EE decreases with increasing $R_{u}^{\mathrm{min}}$ and the number of SUEs in Figures 4 and 5, respectively. The reason is that interference increases in dense small cell networks.

\section{Conclusions}

In this paper, we have firstly established EE maximization problem of joint user association and power allocation considering minimum data rate requirement of SUEs and maximum transmit power constraint of SBSs. Then, a dynamic CoMP-JT algorithm has been proposed to solve the formulated problem. In the first phase, SUEs have been associated with the SBSs close to them within the communication range to reduce the loss of power. In the second phase, through the methods of fractional programming and successive convex approximation, the EE maximization problem has been transformed into a convex problem that was solved by the given power allocation optimization algorithm. Moreover, we have analyzed the fact that the proposed dynamic CoMP-JT solution has a much lower computational complexity than the optimal solution. In the simulation, the performance of the proposed solution has been compared with nearoptimal, SecondNear-CoMP, no-CoMP, and random-CoMP solutions, which demonstrate that the proposed algorithm has a better performance.

\section{Data Availability}

The data is based on our established network model. 


\section{Conflicts of Interest}

The authors declare that there are no conflicts of interest regarding the publication of this paper.

\section{Acknowledgments}

This paper is supported in part by the National Natural Science Foundation of China [nos. 91638202, 61231008, and 61571351].

\section{References}

[1] J. G. Andrews, S. Buzzi, and W. Choi, "What will 5G be?" IEEE Journal on Selected Areas in Communications, vol. 32, no. 6, pp. 1065-1082, 2014.

[2] A. Gupta and R. K. Jha, "A survey of 5G network: architecture and emerging technologies," IEEE Access, vol. 3, pp. 1206-1232, 2015.

[3] C.-X. Wang, F. Haider, X. Gao et al., "Cellular architecture and key technologies for $5 \mathrm{G}$ wireless communication networks," IEEE Communications Magazine, vol. 52, no. 2, pp. 122-130, 2014.

[4] N. Lee and R. W. Heath, "Advanced interference management technique: Potentials and limitations," IEEE Wireless Communications Magazine, vol. 23, no. 3, pp. 30-38, 2016.

[5] A. H. Jafari, D. Lopez-Perez, M. Ding, and J. Zhang, "Study on Scheduling Techniques for Ultra Dense Small Cell Networks," in Proceedings of the 2015 IEEE 82nd Vehicular Technology Conference (VTC Fall), pp. 1-6, Boston, MA, USA, September 2015.

[6] X. Wang, Y. Wang, and H. Zhu, "Energy-efficient multi-job scheduling model for cloud computing and its genetic algorithm," Mathematical Problems in Engineering, vol. 2012, Article ID 589243, 16 pages, 2012.

[7] E. Pateromichelakis, M. Shariat, A. Quddus, M. Dianati, and R. Tafazolli, "Dynamic clustering framework for multi-cell scheduling in dense small cell networks," IEEE Communications Letters, vol. 17, no. 9, pp. 1802-1805, 2013.

[8] Q. Cui, H. Wang, P. Hu et al., "Evolution of limited-feedback CoMP systems from $4 \mathrm{G}$ to 5G: CoMP features and limitedfeedback approaches," IEEE Vehicular Technology Magazine, vol. 9, no. 3, pp. 94-103, 2014.

[9] M. Feng, X. She, L. Chen, and Y. Kishiyama, "Enhanced dynamic cell selection with muting scheme for DL CoMP in LTE-A," in Proceedings of the 2010 IEEE 71st Vehicular Technology Conference, VTC 2010-Spring, Taiwan, May 2010.

[10] X. Li, Q. Cui, Y. Liu, and X. Tao, "An effective scheduling scheme for CoMP in heterogeneous scenario," in Proceedings of the 2012 IEEE 23rd International Symposium on Personal, Indoor and Mobile Radio Communications, PIMRC 2012, pp. 870-874, Australia, September 2012.

[11] G. Nigam, P. Minero, and M. Haenggi, "Coordinated multipoint joint transmission in heterogeneous networks," IEEE Transactions on Communications, vol. 62, no. 11, pp. 4134-4146, 2014.

[12] J. Zhao, T. Q. S. Quek, and Z. Lei, "Coordinated multipoint transmission with limited backhaul data transfer," IEEE Transactions on Wireless Communications, vol. 12, no. 6, pp. 27622775, 2013.

[13] D. W. K. Ng, E. S. Lo, and R. Schober, "Energy-efficient resource allocation in multi-cell OFDMA systems with limited backhaul capacity," IEEE Transactions on Wireless Communications, vol. 11, no. 10, pp. 3618-3631, 2012.

[14] K. M. S. Huq, S. Mumtaz, J. Bachmatiuk, J. Rodriguez, X. Wang, and R. L. Aguiar, "Green HetNet CoMP: Energy Efficiency Analysis and Optimization," IEEE Transactions on Vehicular Technology, vol. 64, no. 10, pp. 4670-4683, 2015.

[15] B. Du, C. Pan, W. Zhang, and M. Chen, "Distributed energyefficient power optimization for CoMP systems with max-min fairness," IEEE Communications Letters, vol. 18, no. 6, pp. 9991002, 2014.

[16] S. Kim and C. Cho, "Power efficient CoMP-joint transmission with two stage QoSs," Transactions on Emerging Telecommunications Technologies, vol. 28, no. 4, p. e3014, 2017.

[17] R. Sun, M. Hong, and Z. Luo, "Joint downlink base station association and power control for max-min fairness: computation and complexity," IEEE Journal on Selected Areas in Communications, vol. 33, no. 6, pp. 1040-1054, 2015.

[18] G. Ye, H. Zhang, H. Liu et al., "Energy efficient joint user association and power allocation in a two-tier heterogeneous network," in IEEE Global Communications Conference, pp. 1-5, 2016.

[19] Y. Li, M. Sheng, C. Yang, and X. Wang, "Energy efficiency and spectral efficiency tradeoff in interference-limited wireless networks," IEEE Communications Letters, vol. 17, no. 10, pp. 1924-1927, 2013.

[20] S. Boyd and L. Vandenberghe, Convex Optimization, Cambridge University Press, UK, 2004.

[21] E. U. T. R. Access, "Further advancements for E-UTRA physical layer aspects," Tech. Rep. 3GPP TR 36, 2010. 


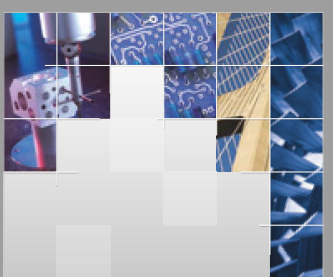

\section{Enfincering}
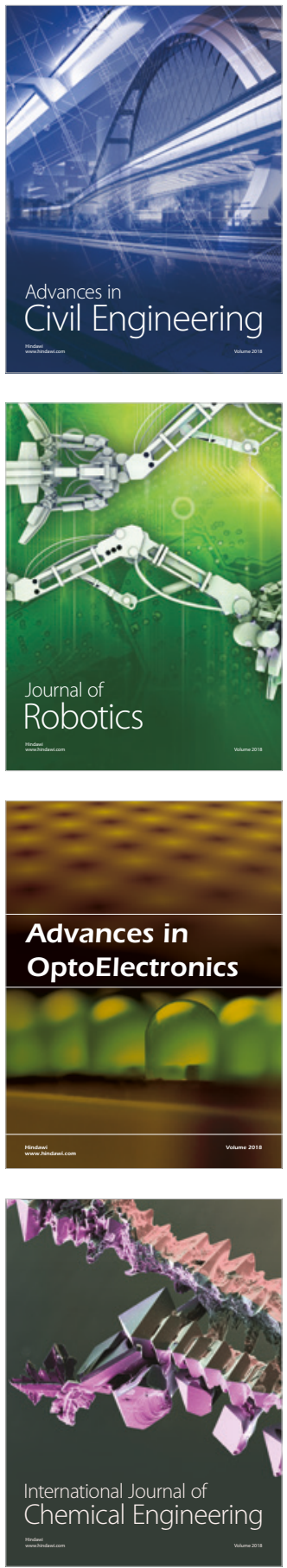

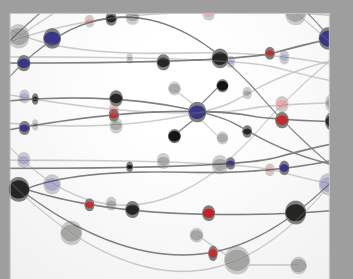

\section{Rotating \\ Machinery}

The Scientific World Journal

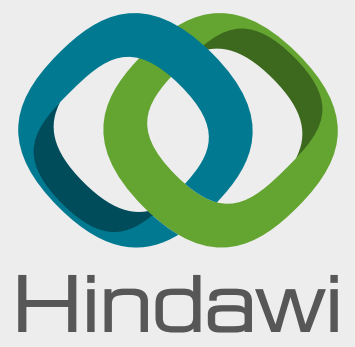

Submit your manuscripts at

www.hindawi.com
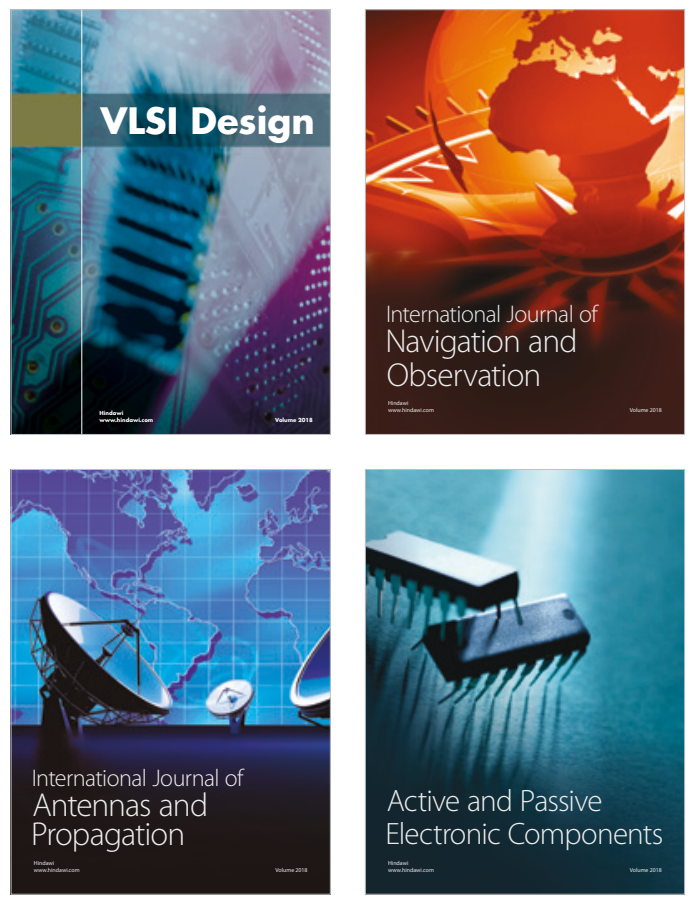
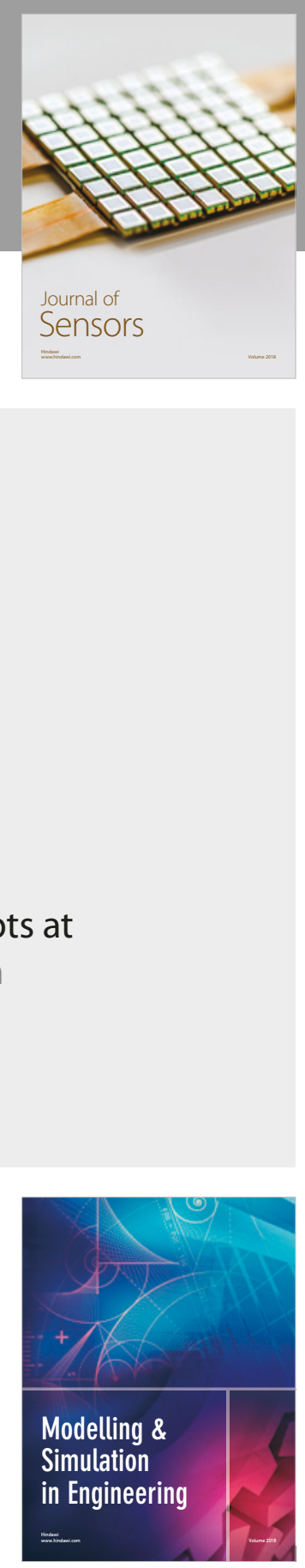

\section{Advances \\ Multimedia}
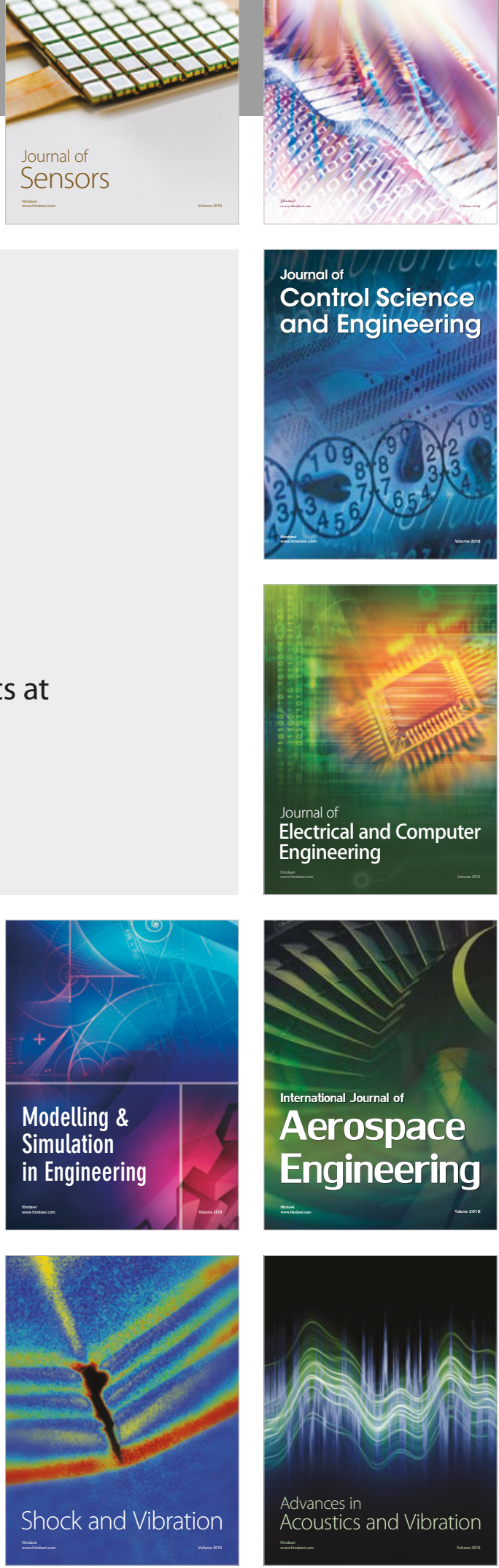